\title{
THE EFFECTS OF SURFACTANT SOLUBILITY AND CONCENTRATION-DEPENDANT FLUID DENSITY ON THE STABILITY OF INCLINED THIN FILM FLOWS
}

\author{
by \\ Flavio Firmino Lunda \\ Bachelor of Science in Medical Physics, Ryerson, 2014
}

\author{
A thesis \\ presented to Ryerson University \\ in partial fulfillment of the \\ requirements for the degree of \\ Master of Science \\ in the Program of \\ Applied Mathematics
}

Toronto, Ontario, Canada, 2016

(C)Flavio Firmino Lunda 2016 


\section{Author's Declaration}

I hereby declare that I am the sole author of this thesis. This is a true copy of the thesis, including any required final revisions, as accepted by my examiners.

I authorize Ryerson University to lend this thesis to other institutions or individuals for the purpose of scholarly research.

I further authorize Ryerson University to reproduce this thesis by photocopying or by other means, in total or in part, at the request of other institutions or individuals for the purpose of scholarly research.

I understand that my thesis may be made electronically available to the public. 


\begin{abstract}
The effects of surfactant solubility and concentration-dependant fluid density on the stability of inclined thin film flows

Master of Science 2016

Flavio Firmino Lunda

Applied Mathematics

Ryerson University

An analytical investigation of the stability of a surfactant-laden liquid film with a free surface, flowing under the influence of gravity on an inclined plane, is conducted. This investigation focusses on the onset of instability of a steady equilibrium flow when the surfactant concentration along the surface affects surface tension, while at the same time, the concentration of surfactant in the bulk of the layer affects the mass density of the liquid. A linear stability analysis is carried out and an asymptotic solution is obtained for the growth rate of long perturbations. The results obtained reflect how the various control parameters influence the onset of instability.
\end{abstract}




\section{Acknowledgements}

I would like to dedicate this page to thank the various people who have helped me navigate through the resources and the process of completing this thesis work. To my research supervisor, Dr. Jean-Paul Pascal, I honestly have no words to describe how much I look up to you. You have been immensely generous and infinitely resourceful. Thank you very much for providing me the guidance and passing on the knowledge that I needed to complete this work. I could not have been in better hands, and my gratitude to you will carry on forever. I also would like to acknowledge my friends and classmates for the great two years that we spent together, it was a great journey for me, and I learned a lot from all of you. A special word of thanks to my dear friend Eglal Ellaban who not only played a pivotal role in helping me fill some of the conceptual gaps in the beginning of my work, but also inspired me to work harder with her personal story of survival and hard work as a single mother of four. Last but not least,

thank you to the Department of Mathematics at Ryerson University for giving me the opportunity to acquire my Masters Degree and for such a great educational experience. 


\section{Dedication}

The day that I started working on this page, I learned that you passed away. My heart is broken, but I am filled with strength and determination because you dreamed of this for me. So, the best I can do is pick up the pieces and continue on the journey, since you might be watching and cheering from eternity. Thank you Uncle João Mauno for believing in me and giving me the confidence to know that can be or do anything I want. I dedicate this thesis to you and the great woman who I had the pleasure to call Mother, Alice Felizarda Firmino. Thank you both for shaping me into who I am today. I hope you are watching down and receiving this honour from where you are. 


\section{Contents}

1 Introduction $\quad 1$

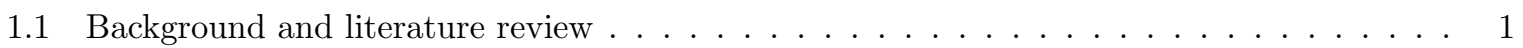

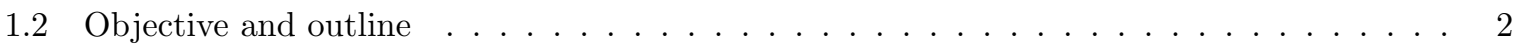

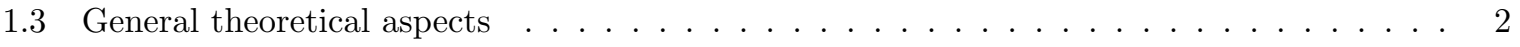

2 Mathematical Analysis $\quad 4$

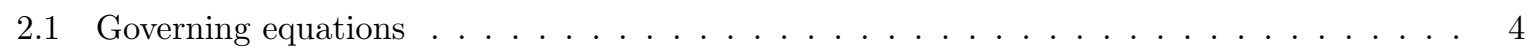

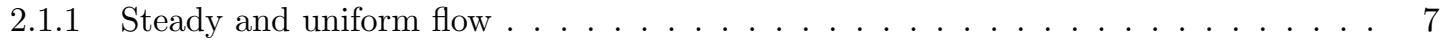

2.2 Linear stability analysis $\ldots \ldots \ldots \ldots \ldots \ldots \ldots \ldots$

$2.2 .1 \quad$ Asymptotic Analysis . . . . . . . . . . . . . . . . . . . . . 11

3 Results $\quad 17$

3.1 Interpretation and discussion of the results $\ldots \ldots \ldots \ldots$

4 Conclusion $\quad 25$

$\begin{array}{ll}\text { References } & 27\end{array}$ 


\section{List of Figures}

2.1 Schematic representation of a thin film flowing down an inclined plane . . . . . . . . . 4

3.1 The critical Reynolds number as a function of the equilibrium surface concentration for various values of $\xi_{s}$ with $\cot \beta=1, K a=100$ and $\Sigma=2 \ldots \ldots$. . . . . . . . 18

3.2 The critical Reynolds number as a function of the equilibrium surface concentration for various values of $\alpha$ with $\cot \beta=1, K a=100$ and $\Sigma=2, S c_{b}=700, S c_{s}=700, k_{s}=1$

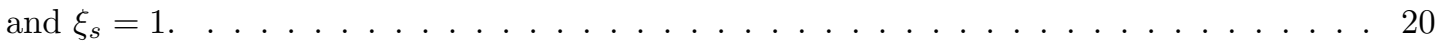

3.3 The critical Reynolds number as a function of the equilibrium surface concentration for various values of $S c_{b}$ with $\cot \beta=1, K a=100$ and $\Sigma=2, \alpha=0.006, S c_{s}=700, k_{s}=1$

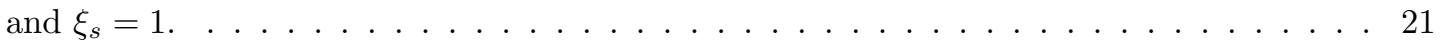

3.4 The critical Reynolds number as a function of the equilibrium surface concentration for various values of $\cot \beta$ with $K a=100$ and $\Sigma=2, \alpha=0.006, S c_{s}=700, S c_{b}=700$,

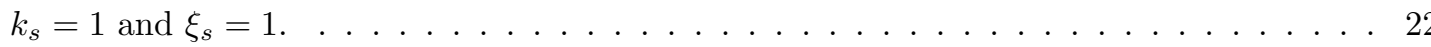

3.5 The critical Reynolds number as a function of the equilibrium surface concentration for various values of $K a$ with $\cot \beta=1$ and $\Sigma=2, \alpha=0.006, S c_{s}=700, S c_{b}=700, k_{s}=1$

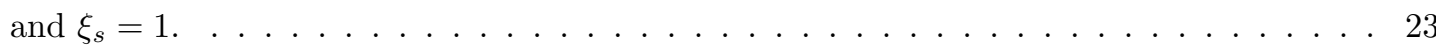

3.6 The critical Reynolds number as a function of the equilibrium surface concentration for various values of $\Sigma$ with $\cot \beta=1$ and $K a=100, \alpha=0.006, S c_{s}=700, S c_{b}=700$,

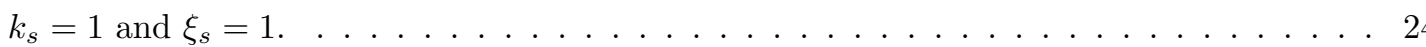




\section{Chapter 1}

\section{Introduction}

\subsection{Background and literature review}

The formation of propagating waves along an inclined free surface, as a model for interfacial instability, was established by Kapitza and Kapitza in their experiments with the gravity driven flow of uncontaminated thin fluid films [1]. Since then, numerous theoretical investigations have been performed to predict the critical conditions for the onset of instability. Benjamin [2] and Yih [3], were the first to accomplish this, by linearizing the governing equations, and asymptotically expanding them in powers of the wavenumber, as a small parameter. Such an approximation is accurate due to the fact that long-wave perturbations are the most unstable and are responsible for the onset of instability in the flow [1].

The major contributor to the instability of the inclined flow of a fluid layer with uniform thickness is inertia. If sufficient, inertia amplifies perturbations in the thickness of the layer leading to the formation of large amplitude waves propagating along the surface. Another important factor is the Marangoni effect: Variation in surface tension generates so called Marangoni stresses which cause fluid flow along the surface towards regions of higher surface tension.

Variation in surface tension can be caused by the presence of chemical agents referred to as surfactants. Surfactants are surface-active compounds that lower the surface tension, and are known to significantly affect interfacial instabilities in falling fluid films. It was first demonstrated by Benjamin [4] and Whitaker [5] that if the surfactants are insoluble, i.e. the entire quantity is absorbed by the surface and does not dissolve into the bulk, then they act to stabilize the flow. Later theoretical investigations include the work of Blyth \& Pozrikidis [6], Pereira et al. [7] and Pereira \& Kalliadasis [8]. Laboratory observations also indicate the stabilizing effect on liquid film flows when doping them with even small amounts of surfactants. Some of the experiments that prove the dampening of waves by surfactants, include the investigations by Emmert \& Pigford [9], Stirba \& Hurt [10], and Tailby \& Portalsky [11].

It turns out that most surfactants are soluble to some extent. In order to capture this effect, a theoretical model must include an equation for the bulk concentration of surfactant, and couple it to the equation for the surface concentration by capturing the absorption/desorption fluxes between the 
surface and the bulk of the liquid layer. Karapetsas and Bontozoglou $[12,13]$ have recently studied the stability of inclined flow contaminated with a soluble surfactant. They found that surfactant solubility enhances the flow instability.

\subsection{Objective and outline}

The study undertaken in this thesis is motivated by the expectation that the presence of dissolved surfactant in the bulk of the liquid layer will affect its mass density. We thus implement a mathematical model for surfactant-laden film flow, i.e. with the case of soluble surfactant, with a variable mass density which is a function of the solute concentration.

The remainder of this chapter provides an introduction to some general theoretical aspects relevant to our topic, such as the flow equations for a fluid with variable properties and general features of the constitutive relations for surface tension and mass density. In chapter 2 we derive the governing equations and obtain a simple equilibrium solution corresponding to a steady flow that is uniform in the stream wise direction of the flow. We then perform a linear stability analysis of this equilibrium flow, obtaining asymptotic approximations based on the assumption of perturbations with small wavenumber. In chapter 3 we present and interpret our results, and finally, in chapter 4 we summarize our investigation.

\subsection{General theoretical aspects}

In general, the governing equations for the two-dimensional inclined flow of a Newtonian fluid with variable properties (viscosity and mass density) are given as the following [14]

$$
\begin{gathered}
\frac{D \rho}{D t}+\rho\left(\frac{\partial u}{\partial x}+\frac{\partial w}{\partial z}\right)=0 \\
\rho \frac{D u}{D t}=-\frac{\partial p}{\partial x}+g \rho \sin \beta+\frac{\partial}{\partial x}\left[2 \mu \frac{\partial u}{\partial x}-\frac{2}{3} \mu\left(\frac{\partial u}{\partial x}+\frac{\partial w}{\partial z}\right)\right]+\frac{\partial}{\partial z}\left[\mu\left(\frac{\partial u}{\partial z}+\frac{\partial w}{\partial x}\right)\right] \\
\rho \frac{D w}{D t}=-\frac{\partial p}{\partial z}-g \rho \cos \beta+\frac{\partial}{\partial z}\left[2 \mu \frac{\partial w}{\partial z}-\frac{2}{3} \mu\left(\frac{\partial u}{\partial x}+\frac{\partial w}{\partial z}\right)\right]+\frac{\partial}{\partial x}\left[\mu\left(\frac{\partial u}{\partial z}+\frac{\partial w}{\partial x}\right)\right] .
\end{gathered}
$$

Equation 1.1 is the conservation of mass equation and equations 1.2 and 1.3 are the Navier-Stokes equations for the momentum conservation along the $x$-direction and the $z$-direction respectively. The notation $\frac{D}{D t}=\frac{\partial}{\partial t}+u \frac{\partial}{\partial x}+w \frac{\partial}{\partial z}$ represents the material derivative operator. Also, $\rho$ denotes the mass density of the fluid, while $u=u(x, z, t)$ and $w=(x, z, t)$ denote the fluid's velocity components in the $x$-direction and the $z$-direction, respectively. Additionally, $p$ denotes the pressure, $g$ denotes the acceleration due to gravity, $\mu$ denotes the dynamic viscosity and $\beta$ is the inclination angle.

For the purpose of our study, we require an equation of the concentration of surfactant in the bulk. We will use the following conservation of mass equation, which accounts for advective and diffusive transport of a contaminant dissolved in a flowing fluid 


$$
\frac{D \phi}{D t}=D_{b}\left(\frac{\partial^{2} \phi}{\partial x^{2}}+\frac{\partial^{2} \phi}{\partial z^{2}}\right)
$$

where $\phi=\phi(x, z, t)$ is the concentration of contaminant, and $D_{b}$ denotes the mass diffusivity in the fluid.

For problems with variable mass density, it is customary to resort to a linear constitutive relation. Accordingly, for our investigation we will assume that the dependence of density on surfactant concentration is given by

$$
\rho=\rho_{0}(1+\hat{\alpha} \phi)
$$

where, $\rho_{0}$ is the density of the clean liquid without surfactant and $\hat{\alpha}$ is the solutal expansion coefficient. In our investigation we are assuming that the density of the solution increases as more surfactant dissolves in it, therefore we consider $\hat{\alpha}$ to be a nonnegative parameter.

In general, surface tension is a function of the concentration of surfactant absorbed on the surface. However, since our focus is a linear stability analysis we will directly consider the linearized formulation. Linearizing about $\Gamma=\Gamma_{E}$ we have

$$
\gamma(\Gamma)=\gamma\left(\Gamma_{E}\right)+\left.\frac{d \gamma}{d \Gamma}\right|_{\Gamma=\Gamma_{E}}\left(\Gamma-\Gamma_{E}\right)
$$

where $\gamma$ is the surface tension, $\Gamma$ is the surface concentration of surfactant and $\Gamma_{E}$ is a reference value, which, in our analysis will be the value for the equilibrium solution. In most cases surface tension decreases with surfactant concentration, so we have

$$
\left.\frac{d \gamma}{d \Gamma}\right|_{\Gamma=\Gamma_{E}} \leq 0 .
$$

This quantity gives the rate of change of the surface tension and thus measures the significance of the Marangoni effect. We point out that in order to determine the explicit dependence on the equilibrium concentration a specific constitutive relation must be considered. 


\section{Chapter 2}

\section{Mathematical Analysis}

\subsection{Governing equations}

Figure 2.1 shows the flow configuration of a thin layer of fluid flowing down an impermeable inclined plane under the influence of gravity. The plane is inclined at an angle $\beta$ with the horizontal. The fluid is assumed to be Newtonian and the flow is assumed to be two-dimensional and laminar. An $x-z$ coordinate system is defined so that the $x$-axis points along the plane in the downhill direction and the $z$-axis points into the fluid. Meanwhile, the thickness of the fluid layer is given by $h(x, t)$.

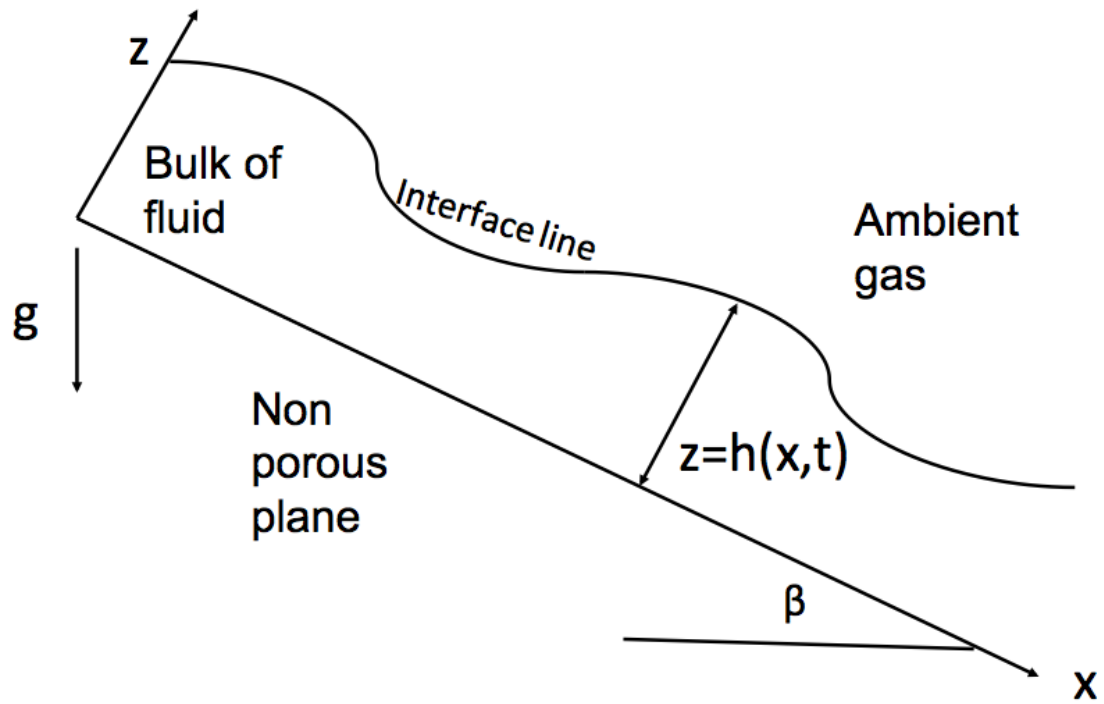

Figure 2.1: Schematic representation of a thin film flowing down an inclined plane

As it has been explained, in this work we assume the fluid to have constant viscosity, but variable density. In order to obtain the equations of motion we apply the Boussinesq approximation whereby the 
density is taken to be constant except when it is multiplied by the gravitational acceleration. Therefore, the dimensioned governing equations become as follows

$$
\begin{gathered}
\frac{\partial u}{\partial x}+\frac{\partial w}{\partial z}=0 \\
\rho_{0} \frac{D u}{D t}=-\frac{\partial p}{\partial x}+\mu\left(\frac{\partial^{2} u}{\partial x^{2}}+\frac{\partial^{2} u}{\partial z^{2}}\right)+\rho g \sin \beta \\
\rho_{0} \frac{D w}{D t}=-\frac{\partial p}{\partial z}+\mu\left(\frac{\partial^{2} w}{\partial x^{2}}+\frac{\partial^{2} w}{\partial z^{2}}\right)-\rho g \cos \beta
\end{gathered}
$$

where the notation is consistent with that for equations $1.1-1.3$ and $\rho=\rho_{0}(1+\hat{\alpha} \phi)$ with $\phi$ being the concentration of surfactants in the bulk and $\hat{\alpha}$ the solutal expansion coefficient. To these we append the equations for the surfactant concentration in the bulk

$$
\frac{\partial \phi}{\partial t}+u \frac{\partial \phi}{\partial x}+w \frac{\partial \phi}{\partial z}=D_{b}\left(\frac{\partial^{2} \phi}{\partial x^{2}}+\frac{\partial^{2} \phi}{\partial z^{2}}\right)
$$

where $D_{b}$ is the mass diffusivity.

We now turn to establishing boundary conditions at the interfaces of the film: the surface of the fluid layer, which is the interface with the ambient gas, and the bottom of the layer which is interface with the substrate (the solid plane on which the fluid is flowing).

At the surface of the liquid film, located at $z=h(x, t)$, the normal stress exerted by the flow is balanced by the pressure in the ambient gas and that is due to the surface tension. The corresponding condition is expressed as

$$
p=p_{a i r}+\frac{2 \mu}{1+\frac{\partial h^{2}}{\partial x}}\left[\frac{\partial h^{2}}{\partial x} \frac{\partial u}{\partial x}+\frac{\partial w}{\partial z}-\frac{\partial h}{\partial x}\left(\frac{\partial u}{\partial z}+\frac{\partial w}{\partial x}\right)\right]-\frac{\gamma{\frac{\partial^{2} h}{\partial x^{2}}}^{3 / 2}}{\left(1+\frac{\partial h^{2}}{\partial x}\right)^{3 / 2}}
$$

where $p_{a i r}$ is the pressure in the ambient gas and $\gamma$ is the surface tension given by equation 1.6.

On the other hand, the tangential stress exerted by the flow at the surface is balanced by the Marangoni stress due to the variation in surface tension. So, at $z=h(x, t)$ we have

$$
\left(\frac{\partial \gamma}{\partial x}+\frac{\partial h}{\partial x} \frac{\partial \gamma}{\partial z}\right) \sqrt{1+\left(\frac{\partial h}{\partial x}\right)^{2}}=\mu\left[\left(1-\frac{\partial h^{2}}{\partial x}\right)\left(\frac{\partial w}{\partial x}+\frac{\partial u}{\partial z}\right)-4\left(\frac{\partial h}{\partial x}\right)\left(\frac{\partial u}{\partial x}\right)\right] .
$$

If it is assumed that the evaporation of the liquid is negligible, then a kinematic condition can be applied which requires that the normal velocity of the fluid at the surface equals the normal velocity of the surface. The formulation is

$$
w=\frac{\partial h}{\partial t}+u \frac{\partial h}{\partial x} \quad \text { at } \quad z=h(x, t)
$$

For the conservation of surfactant absorbed by the surface we use the following advection-diffusion- 
reaction equation $[15]$

$$
\begin{aligned}
\frac{\partial \Gamma}{\partial t}+u \frac{\partial \Gamma}{\partial x}+\frac{\Gamma}{1+\left(\frac{\partial h}{\partial x}\right)^{2}}\left[\frac{\partial u}{\partial x}+\left(\frac{\partial h}{\partial x}\right)\left(\frac{\partial w}{\partial x}\right)+\frac{\partial h}{\partial x}\left(\frac{\partial u}{\partial z}+\frac{\partial h}{\partial x} \frac{\partial w}{\partial z}\right)\right]= & \frac{D_{s}}{\sqrt{1+\left(\frac{\partial h}{\partial x}\right)^{2}}} \frac{\partial}{\partial x}\left(\frac{\frac{\partial \Gamma}{\partial x}}{\sqrt{1+\left(\frac{\partial h}{\partial x}\right)^{2}}}\right)+J_{b a}
\end{aligned}
$$

where $\Gamma(x, t)$ is the surface concentration of surfactant, $D_{s}$ is the molecular diffusivity on the surface and $J_{b a}$ denotes the net flux of absorption of surfactant from the bulk to the surface. Now, the desorption flux is proportional to $\Gamma$ while the absorption flux is proportional to $\left(1-\frac{\Gamma}{\Gamma_{\infty}}\right) \phi$, where $\Gamma_{\infty}$ is the maximum concentration that the surface can hold. Consequently, we have

$$
J_{b a}=k_{1}\left(1-\frac{\Gamma}{\Gamma_{\infty}}\right) \phi-k_{2} \Gamma, \quad \text { at } \quad z=h(x, t)
$$

where $k_{1}$ and $k_{2}$ are nonnegative proportionality constants referred to as the absorption reaction rate and the desorption reaction rate respectively.

Of course, the surfactant flux to the surface must equal the flux from the bulk of the fluid. By Fick's law, the diffusive flux is proportional to the concentration gradient leading to the following condition at $z=h(x, t)$

$$
J_{b a}=\frac{D_{b}}{\sqrt{1+\left(\frac{\partial h}{\partial x}\right)^{2}}}\left(\frac{\partial h}{\partial x} \frac{\partial \phi}{\partial x}-\frac{\partial \phi}{\partial z}\right) .
$$

At the impermeable substrate we apply the no-slip and no-penetration conditions, as well as requiring that the normal surfactant flux is zero. We thus have the following conditions at $z=0$

$$
u=w=\frac{\partial \phi}{\partial z}=0
$$

We now scale the variables and nondimensionalize the equations. We scale the space variables by

$$
H=\left(\frac{3 \mu Q}{g \rho_{0} \sin \beta}\right)^{1 / 3}
$$

which is the thickness of the equilibrium flow of clean liquid generated by a prescribed flow rate $Q$. The surface concentration is scaled by the maximal value, $\Gamma_{\infty}$ while the bulk concentration is scaled by $\frac{\Gamma_{\infty}}{H}$. Finally, $\frac{\rho_{0} Q^{2}}{H^{2}}$ is used as the pressure scale. Specifically, we apply the following transformation

$$
(x, z)=H\left(x^{\star}, z^{\star}\right), h=H h^{\star},(u, w)=\frac{Q}{H}\left(u^{\star}, w^{\star}\right), t=\frac{H^{2}}{Q} t^{\star}, p-p_{a i r}=\frac{\rho_{0} Q^{2}}{H^{2}} p^{\star}, \Gamma=\Gamma_{\infty} \Gamma^{\star}, \phi=\frac{\Gamma_{\infty}}{H} \phi^{\star} .
$$

The transformed equations then are, dropping the stars for notational convenience,

$$
\frac{\partial u}{\partial x}+\frac{\partial w}{\partial z}=0
$$




$$
\begin{gathered}
R e \frac{D u}{D t}=-\operatorname{Re} \frac{\partial p}{\partial x}+\frac{\partial^{2} u}{\partial x^{2}}+\frac{\partial^{2} u}{\partial z^{2}}+3(1+\alpha \phi) \\
R e \frac{D w}{D t}=-R e \frac{\partial p}{\partial z}+\frac{\partial^{2} w}{\partial x^{2}}+\frac{\partial^{2} w}{\partial z^{2}}-3 \cot \beta(1+\alpha \phi) \\
\frac{\partial \phi}{\partial t}+u \frac{\partial \phi}{\partial x}+w \frac{\partial \phi}{\partial z}=\frac{1}{P e_{b}}\left(\frac{\partial^{2} \phi}{\partial x^{2}}+\frac{\partial^{2} \phi}{\partial z^{2}}\right)
\end{gathered}
$$

where $\alpha=\frac{\hat{\alpha} \Gamma_{\infty}}{H}, P e_{b}=\frac{Q}{D_{b}}$ and $R e=\frac{\rho_{0} Q}{\mu}$ is the Reynolds number.

Next we list the scaled interface conditions. At $z=h(x, t)$ we get

$$
p=\frac{2}{R e\left(1+\frac{\partial h^{2}}{\partial x}\right)}\left[\frac{\partial h^{2}}{\partial x} \frac{\partial u}{\partial x}+\frac{\partial w}{\partial z}-\frac{\partial h}{\partial x}\left(\frac{\partial u}{\partial z}+\frac{\partial w}{\partial z}\right)\right]-\frac{\frac{\partial^{2} h}{\partial x^{2}}}{\left(1+\frac{\partial h^{2}}{\partial x}\right)^{3 / 2}}\left[W e-M\left(\Gamma-\Gamma_{E}\right)\right]
$$

where $W e=\frac{\gamma\left(\Gamma_{E}\right) H}{\rho_{0} Q^{2}}$ and $M=-\left.\frac{H}{\rho_{0} Q^{2}} \frac{d \gamma}{d \Gamma}\right|_{\Gamma=\Gamma_{E}}$,

$$
\begin{aligned}
-M R e \frac{\partial \Gamma}{\partial x} \sqrt{1+\frac{\partial h^{2}}{\partial x}} & =\left(1-\frac{\partial h^{2}}{\partial x}\right)\left(\frac{\partial w}{\partial x}+\frac{\partial u}{\partial z}\right)-4 \frac{\partial h}{\partial x} \frac{\partial u}{\partial x}, \\
w & =\frac{\partial h}{\partial t}+u \frac{\partial h}{\partial x}, \\
\frac{\partial \Gamma}{\partial t}+\frac{\partial \Gamma}{\partial x} u+\frac{\Gamma}{1+\frac{\partial h^{2}}{\partial x}}\left[\frac{\partial u}{\partial x}+\frac{\partial h}{\partial x} \frac{\partial w}{\partial x}+\frac{\partial h}{\partial x}\left(\frac{\partial u}{\partial z}+\frac{\partial h}{\partial x} \frac{\partial w}{\partial z}\right)\right] & =\frac{1}{P e_{s}} \frac{1}{\sqrt{1+\frac{\partial h^{2}}{\partial x}} \frac{\partial}{\partial x}\left(\frac{\frac{\partial \Gamma}{\partial x}}{\sqrt{1+\frac{\partial h^{2}}{\partial x}}}\right)+k_{s}\left[\xi_{s}(1-\Gamma) \phi-\Gamma\right]}
\end{aligned}
$$

where $P e_{s}=\frac{Q}{D_{s}}, k_{s}=\frac{H k_{2}}{U}$ and $\xi_{s}=\frac{k_{1}}{k_{2} H}$, and finally,

$$
\frac{1}{P e_{b} \sqrt{1+\frac{\partial h^{2}}{\partial x}}}\left(\frac{\partial h}{\partial x} \frac{\partial \phi}{\partial x}-\frac{\partial \phi}{\partial z}\right)=k_{s}\left[\xi_{s}(1-\Gamma) \phi-\Gamma\right] .
$$

At $z=0$ the conditions are

$$
u=w=\frac{\partial \phi}{\partial z}=0
$$

\subsubsection{Steady and uniform flow}

Our model admits a simple solution independent of $x$ and $t$ given by

$$
\Gamma=\Gamma_{E}=\text { const. }, \quad \phi=\phi_{E}=\frac{\Gamma_{E}}{\xi_{s}\left(1-\Gamma_{E}\right)},
$$




$$
h=1, \quad w \equiv 0, \quad u=u_{E}(z) \equiv 3\left(1+\alpha \phi_{E}\right)\left(z-\frac{z^{2}}{2}\right), \quad p=p_{E}(z) \equiv-\frac{3 \cot \beta}{R e}\left(1+\alpha \phi_{E}\right)(z-1),
$$

where $\Gamma_{E}$ is an arbitrary constant. This solution corresponds to a steady flow that is uniform in the streamwise direction, i.e. with distance along the incline. The control parameters for this flow are $\Gamma_{E}$, the surface concentration of surfactant, $\alpha$, the scaled solutal expansion coefficient, $\xi_{s}$, the ratio of absorption to desorption rates which provides a measure of the solubility of the surfactant (the insoluble case corresponding to $\left.\xi_{s} \rightarrow \infty\right), \beta$, the inclination of the flow and $R e$, the Reynolds number which controls the flow rate.

\section{$2.2 \quad$ Linear stability analysis}

Our task now is to determine the stability of the steady solution obtained above, to which we will hence refer to from here on as the "equilibrium solution". To this end we carry out a linear stability analysis. Accordingly, we consider the perturbed equilibrium solution given by $u=u_{E}+\tilde{u}(x, z, t), w=\tilde{w}(x, z, t)$, $\phi=\phi_{E}+\tilde{\phi}(x, z, t), p=p_{E}+\tilde{p}(x, z, t), \Gamma=\Gamma_{E}+\tilde{\Gamma}(x, t), h=1+\tilde{h}(x, t)$, where the perturbations are denoted by tilde. The perturbations are assumed to be infinitesimal, therefore, upon substituting the perturbed equilibrium into the problem we linearize with respect to the perturbations. We thus obtain the following equations

$$
\begin{gathered}
\frac{\partial \tilde{u}}{\partial x}+\frac{\partial \tilde{w}}{\partial z}=0 \\
\operatorname{Re}\left[\frac{\partial \tilde{u}}{\partial t}+u_{E}(z) \frac{\partial \tilde{u}}{\partial x}+\tilde{w} \frac{d u_{E}}{d z}\right]=-\operatorname{Re} \frac{\partial \tilde{p}}{\partial x}+\frac{\partial^{2} \tilde{u}}{\partial x^{2}}+\frac{\partial^{2} \tilde{u}}{\partial z^{2}}+3 \alpha \tilde{\phi} \\
\operatorname{Re}\left[\frac{\partial \tilde{w}}{\partial t}+u_{E}(z) \frac{\partial \tilde{w}}{\partial x}\right]=-\operatorname{Re} \frac{\partial \tilde{p}}{\partial z}+\frac{\partial^{2} \tilde{w}}{\partial x^{2}}+\frac{\partial^{2} \tilde{w}}{\partial z^{2}}-3 \cot \beta \alpha \tilde{\phi} \\
\frac{\partial \tilde{\phi}}{\partial t}+u_{E}(z) \frac{\partial \tilde{\phi}}{\partial x}=\frac{1}{P e_{b}}\left(\frac{\partial^{2} \tilde{\phi}}{\partial x^{2}}+\frac{\partial^{2} \tilde{\phi}}{\partial z^{2}}\right) .
\end{gathered}
$$

Transferring the conditions at $z=1+\tilde{h}$ to $z=1$ and linearizing leads to the following conditions evaluated at $z=1$

$$
\begin{gathered}
\tilde{p}=\frac{1}{R e} \cot \beta \Phi_{E} \tilde{h}+\frac{2}{R e} \frac{\partial \tilde{w}}{\partial z}-W e \frac{\partial^{2} \tilde{h}}{\partial x^{2}} \\
-M \operatorname{Re} \frac{\partial \tilde{\Gamma}}{\partial x}=\frac{\partial \tilde{w}}{\partial x}+\frac{\partial \tilde{u}}{\partial z}-\Phi_{E} \tilde{h} \\
\frac{\partial \tilde{\Gamma}}{\partial t}+u_{E} \frac{\partial \tilde{\Gamma}}{\partial x}+\Gamma_{E} \frac{\partial \tilde{u}}{\partial x}=\frac{1}{P e_{s}} \frac{\partial^{2} \tilde{\Gamma}}{\partial x^{2}}+k_{s}\left[\xi_{s} \tilde{\phi}\left(1-\Gamma_{E}\right)-\xi_{s} \phi_{E} \tilde{\Gamma}-\tilde{\Gamma}\right]
\end{gathered}
$$




$$
\begin{gathered}
-\frac{1}{P e_{b}} \frac{\partial \tilde{\phi}}{\partial z}=k_{s}\left[\xi_{s} \tilde{\phi}\left(1-\Gamma_{E}\right)-\xi_{s} \phi_{E} \tilde{\Gamma}-\tilde{\Gamma}\right] \\
\tilde{w}=\frac{\partial \tilde{h}}{\partial t}+\frac{\Phi_{E}}{2} \frac{\partial \tilde{h}}{\partial x}
\end{gathered}
$$

where $\Phi_{E}=3\left(1+\alpha \phi_{E}\right)$. At $z=0$ the conditions for the perturbations are

$$
\tilde{\phi}_{z}=\tilde{u}=\tilde{w}=0 .
$$

To further analyze the above linearized equations and boundary conditions, we consider the following normal modes:

$$
\begin{array}{rll}
\tilde{h}(x, t)=\eta e^{i k(x-c t)}, & \tilde{u}(x, z, t)=\hat{u}(z) e^{i k(x-c t)}, & \tilde{w}(x, z, t)=\hat{w}(z) e^{i k(x-c t)}, \\
\tilde{p}(x, z, t)=\hat{p}(z) e^{i k(x-c t)}, \quad \tilde{\phi}=\hat{\phi}(z)(x, z, t) e^{i k(x-c t)} & \tilde{\Gamma}(x, t)=\hat{\Gamma} e^{i k(x-c t)}
\end{array}
$$

where $k$ is a positive real number which coincides with the wavenumber of the perturbation, while $c$ is a complex number, $c=c_{R}+i c_{i}$. The real part, $c_{R}$, equals the phase velocity of the perturbation, and $k c_{i}$, gives the temporal growth rate.

In terms of the normal modes the perturbation equations become

$$
\begin{gathered}
D \hat{w}+i k \hat{u}=0 \\
\operatorname{Re}\left[i k\left(u_{E}-c\right) \hat{u}+D u_{E} \hat{w}\right]=-i k \operatorname{Re} \hat{p}+\left(D^{2}-k^{2}\right) \hat{u}+3 \alpha \hat{\phi} \\
i k \operatorname{Re}\left(u_{E}-c\right) \hat{w}=-\operatorname{Re} D \hat{p}+\left(D^{2}-k^{2}\right) \hat{w}-3 \cot \beta \alpha \hat{\phi} \\
i k\left(u_{E}-c\right) \hat{\phi}=\frac{1}{P e_{b}}\left(D^{2}-k^{2}\right) \hat{\phi}
\end{gathered}
$$

where $D$ denotes the operator $\frac{d}{d z}$. The boundary conditions at $z=1$ become

$$
\begin{gathered}
\hat{p}=\frac{\cot \beta}{R e} \Phi_{E} \eta+\frac{2}{R e} D \hat{w}+W e k^{2} \eta \\
-i k M R e \hat{\Gamma}=i k \hat{w}+D \hat{u}-\Phi_{E} \eta \\
i k\left(\frac{\Phi_{E}}{2}-c\right) \hat{\Gamma}+i k \Gamma_{E} \hat{u}=-\frac{k^{2}}{P e_{s}} \hat{\Gamma}+k_{s}\left[\xi_{s}\left(1-\Gamma_{E}\right) \hat{\phi}-\left(1+\xi_{s} \phi_{E}\right) \hat{\Gamma}\right]
\end{gathered}
$$




$$
-\frac{D \hat{\phi}}{P e_{b}}=k_{s}\left[\xi_{s}\left(1-\Gamma_{E}\right) \hat{\phi}-\left(1+\xi_{s} \phi_{E}\right) \hat{\Gamma}\right]
$$

and

$$
\hat{w}=-i k\left(c-\frac{\Phi_{E}}{2}\right) \eta
$$

At $z=0$ we have

$$
\hat{u}(0)=\hat{w}(0)=D \hat{\phi}(0)=0 .
$$

The next step is to eliminate the pressure from the problem. We begin by combining equations 2.21 and 2.22. Firstly, we apply the $D$ operator to equation 2.21 and multiply equation 2.22 by $-i k$. This gives, respectively,

$$
\operatorname{Re}\left[i k D u_{E} \hat{u}+i k\left(u_{E}-c\right) D \hat{u}+D \hat{w} D u_{E}+\hat{w} D^{2} u_{E}\right]=-i k \operatorname{Re} \hat{p}-k^{2} D \hat{u}+D^{3} \hat{u}+3 \alpha D \hat{\phi}
$$

and

$$
k^{2} \operatorname{Re}\left(u_{E}-c\right) \hat{w}=i k \operatorname{Re} D \hat{p}+i k^{3} \hat{w}-i k D^{2} \hat{w}+3 i k \cot \beta \alpha \hat{\phi} .
$$

Adding these equations yields

$$
\operatorname{Re}\left[i k\left(u_{E}-c\right) D \hat{u}+\hat{w} D^{2} u_{E}+k^{2}\left(u_{E}-c\right) \hat{w}\right]=D^{3} \hat{u}-k^{2} D \hat{u}+i k^{3} \hat{w}-i k D^{2} \hat{w}+3 \alpha(D \hat{\phi}+i k \cot \beta \hat{\phi})
$$

Now we eliminate $\hat{p}(1)$ from the boundary conditions by solving equation 2.21 for $\hat{p}(z)$, substituting in $z=1$, and then substituting the new $\hat{p}(1)$ expression into boundary condition 2.24 :

$$
\operatorname{Re}\left[i k\left(u_{E}-c\right) \hat{u}+\hat{w} D u_{E}\right]+k^{2} \hat{u}-D^{2} \hat{u}+2 i k D \hat{w}-3 \alpha \hat{\phi}=i k\left[k^{2} W e-\cot \beta \Phi_{E}\right] \eta .
$$

Equation 2.20 disappears when the velocity disturbances are expressed in terms of the stream function relations $\tilde{u}=\frac{\partial \psi}{\partial z}$ and $\tilde{w}=-\frac{\partial \psi}{\partial x}$. Therefore, when we apply the normal mode as $\psi=\Psi(z) e^{i k(x-c t)}$, the $z$-dependent factors of the velocity perturbations can be expressed in terms of the stream function as $\hat{u}=D \Psi$ and $\hat{w}=-i k \Psi$. We can thus express the problem in terms of two dependent variables: $\Psi(z)$ and $\hat{\phi}(z)$. From equation 2.29 we obtain an Orr-Sommerfeld type equation:

$$
D^{4} \Psi-\left[i k \operatorname{Re}\left(u_{E}-c\right)+2 k^{2}\right] D^{2} \Psi+\left[i k^{3} \operatorname{Re}\left(u_{E}-c\right)+k^{4}+i k \operatorname{Re} D^{2} u_{E}\right] \Psi+3 \alpha D \hat{\phi}+3 i k \cot \beta \alpha \hat{\phi}=0
$$

while the other differential equation is equation 2.23 expressed as

$$
i k\left(u_{E}-c\right) \hat{\phi}=\frac{1}{P e_{b}}\left(D^{2} \hat{\phi}-k^{2} \hat{\phi}\right) .
$$

The boundary conditions at $z=1$ are obtained from equations 2.30 and $2.25-2.28$, which, in terms of 
the stream function formulation become

$$
\begin{gathered}
D^{3} \Psi-\left[i k R e\left(u_{E}-c\right)+3 k^{2}\right] D \Psi+3 \alpha \hat{\phi}-\left[i k \Phi_{E} \cot \beta+i k^{3} W e\right] \eta=0 \\
-i k M \operatorname{Re} \hat{\Gamma}=D^{2} \Psi+k^{2} \Psi-\Phi_{E} \eta \\
i k\left(\frac{\Phi_{E}}{2}-c\right) \hat{\Gamma}+i k \Gamma_{E} D \Psi=-\frac{k^{2}}{P e_{s}} \hat{\Gamma}+k_{s}\left[\xi_{s}\left(1-\Gamma_{E}\right) \hat{\phi}-\left(1+\xi_{s} \phi_{E}\right) \hat{\Gamma}\right] \\
-\frac{D \hat{\phi}}{P e_{b}}=k_{s}\left[\xi_{s}\left(1-\Gamma_{E}\right) \hat{\phi}-\left(1+\xi_{s} \phi_{E}\right) \hat{\Gamma}\right] \\
-\Psi=\left(\frac{\Phi_{E}}{2}-c\right) \eta .
\end{gathered}
$$

The boundary conditions at $z=0$ are

$$
\Psi(0)=D \Psi(0)=D \hat{\phi}(0)=0 .
$$

The problem given by equations $2.31-2.38$ constitutes an eigenvalue problem with $c$ acting as the parameter that is to be assigned characteristic values. Solving for this quantity will reveal if, for a given set of flow parameters, the perturbation with wavenumber $k$ will be amplified or damped in time.

\subsubsection{Asymptotic Analysis}

We solve the eigenvalue problem $2.31-2.38$ asymptotically as $k \rightarrow 0$. It should be mentioned that the results will only be accurate for determining the stability of very long perturbations. However, we expect these perturbations to be the most unstable, and thus be the ones that trigger instability in the equilibrium flow. Therefore, the asymptotic analysis is sufficient for predicting the onset of instability for the equilibrium flow, which is the goal of this investigation.

We begin the process by expanding all the unknown quantities of the problem in powers of $k$ :

$$
\begin{aligned}
& \Psi=\Psi_{0}(z)+k \Psi_{1}(z)+k^{2} \Psi_{2}(z) \\
& \hat{\phi}=\phi_{0}(z)+k \phi_{1}(z)+k^{2} \phi_{2}(z) \\
& \hat{\Gamma}=\Gamma_{0}+k \Gamma_{1}+k^{2} \Gamma_{2}, \eta=\eta_{0}+k \eta_{1}+k^{2} \eta_{2}, c=c_{0}+k c_{1}+k^{2} c_{2} .
\end{aligned}
$$

The eigenvalue problem can actually be normalized and we can set $\eta_{0}=1$ and $\eta_{1}=\eta_{2}=0$.

Substituting these expansions into the problem and collecting like terms in $k$ leads to a hierarchy of simpler problems which can be solved sequentially. 


\section{The $O(1)$ problem}

Collecting the leading order terms in the differential equations gives

$$
\begin{gathered}
D^{4} \Psi_{0}+3 \alpha D \phi_{0}=0 \\
D^{2} \phi_{0}=0
\end{gathered}
$$

with boundary conditions at $z=0$ given by

$$
D \phi_{0}(0)=\Psi_{0}(0)=D \Psi_{0}(0)=0
$$

and with the following boundary conditions at $z=1$

$$
\begin{gathered}
D^{3} \Psi_{0}+3 \alpha \phi_{0}=0 \\
D^{2} \Psi_{0}=\Phi_{E} \\
c_{0}=\Psi_{0}+\frac{\Phi_{E}}{2} \\
D \phi_{0}=0 \\
\xi_{s}\left(1-\Gamma_{E}\right) \phi_{0}=\left(1+\xi_{s} \phi_{E}\right) \hat{\Gamma}_{0} .
\end{gathered}
$$

Solving this problem we find that $\phi_{0}$ is constant, however the specific value can only be determined when solving the subsequent order problem. The solution for $\Gamma_{0}$ is then obtained from equation 2.44, and, upon expressing $\phi_{E}$ in terms of $\Gamma_{E}$, is given by

$$
\hat{\Gamma}_{0}=\left(1-\Gamma_{E}\right)^{2} \xi_{s} \phi_{0}
$$

Integrating equation 2.39 and using conditions $2.40-2.42$ we obtain the solution for $\Psi_{0}$ :

$$
\Psi_{0}(z)=-\frac{\alpha}{2} \phi_{0} z^{3}+\frac{1}{2}\left(\Phi_{E}+3 \alpha \phi_{0}\right) z^{2}
$$

which we then use in equation 2.43 to get

$$
c_{0}=\Phi_{E}+\alpha \phi_{0} .
$$

This is the leading-order approximation to $c$, however it is real and thus does not offer any information regarding the temporal growth rate of the perturbation. Consequently, we must obtain a higher order term. 


\section{The $O(k)$ problem}

Focusing next on the terms in $k$ we obtain the following differential equations

$$
\begin{gathered}
D^{4} \Psi_{1}-i \operatorname{Re}\left(u_{E}-c_{0}\right) D^{2} \Psi_{0}+i \operatorname{Re} D^{2} u_{E} \Psi_{0}+3 \alpha D \phi_{1}+3 i \cot \beta \alpha \phi_{0}=0 \\
i\left(u_{E}-c_{0}\right) \phi_{0}=\frac{1}{P e_{b}} D^{2} \phi_{1}
\end{gathered}
$$

together with the following boundary conditions

$$
D \phi_{1}(0)=\Psi_{1}(0)=D \Psi_{1}(0)=0
$$

and the following boundary conditions at $z=1$

$$
\begin{gathered}
D^{3} \Psi_{1}-i \operatorname{Re}\left(u_{E}-c_{0}\right) D \Psi_{0}+3 \alpha \phi_{1}-i \Phi_{E} \cot \beta=0 \\
-i M \operatorname{Re} \Gamma_{0}=D^{2} \Psi_{1} \\
i\left(\frac{\Phi_{E}}{2}-c_{0}\right) \Gamma_{0}+i \Gamma_{E} D \Psi_{0}=-\frac{1}{P e_{b}} D \phi_{1} \\
-\frac{D \phi_{1}}{P e_{b}}=k_{s}\left[\xi_{s}\left(1-\Gamma_{E}\right) \phi_{1}-\left(1+\xi_{s} \phi_{E}\right) \Gamma_{1}\right] \\
\Psi_{1}(1)=c_{1} .
\end{gathered}
$$

Integrating equation 2.46 and using the condition $D \phi_{1}(0)=0$ yields

$$
D \phi_{1}=i P e_{b}\left[\Phi_{E}\left(\frac{z^{2}}{2}-\frac{z^{3}}{6}\right)-c_{0} z\right] \phi_{0} .
$$

Then substituting this expression into 2.49 and employing the explicit solutions for $c_{0}$ and $\Gamma_{0}$ from the $O(1)$ problem gives

$$
-\left(\alpha \phi_{0}+\frac{\Phi_{E}}{2}\right)\left(1-\Gamma_{E}\right)^{2} \xi_{s} \phi_{0}+\left(\frac{3 \alpha}{2} \phi_{0}+\Phi_{E}\right) \Gamma_{E}=\left(\alpha \phi_{0}+\frac{2}{3} \Phi_{E}\right) \phi_{0} .
$$

This equation contains $\phi_{0}$ and the control parameters of the problem, and thus provides the solution for $\phi_{0}$ that has not yet been determined. We have obtained the explicit solution using the Maple symbolic algebra software, but the expression is long and complicated and presenting it here serves little purpose.

Integrating equation 2.52 yields

$$
\phi_{1}=i P e_{b}\left[\Phi_{E}\left(\frac{z^{3}}{6}-\frac{z^{4}}{24}\right)-\frac{c_{0}}{2} z^{2}+A\right] \phi_{0}
$$


where the integration constant $A$ is determined by substituting into equation 2.50 :

$$
A=\frac{1}{i P e_{b}\left(1-\Gamma_{E}\right) \xi_{s} \phi_{0}}\left[\frac{i}{k_{s}}\left(\alpha \phi_{0}+\frac{2}{3} \Phi_{E}\right) \phi_{0}+\left(1+\xi_{s} \Phi_{E}\right) \Gamma_{1}\right]+\frac{\alpha}{2} \phi_{0}+\frac{3}{8} \Phi_{E} .
$$

We point out that this solution for $\phi_{1}$ is in terms of $\Gamma_{1}$ which has not yet been determined.

Gathering now the equations that govern $\Psi_{1}$ we have

$$
\begin{aligned}
& D^{4} \Psi_{1}=i \operatorname{Re}\left[u_{E}(z)-c_{0}\right] D^{2} \Psi_{0}-i \operatorname{Re} D^{2} u_{E} \Psi_{0}-3 \alpha D \phi_{1}-3 i \cot \beta \alpha \phi_{0}, \quad 0<z<1 \\
& D^{3} \Psi_{1}(1)=i \operatorname{Re}\left[u_{E}(1)-c_{0}\right] D \Psi_{0}(1)-3 \alpha \phi_{1}(1)+i \Phi_{E} \cot \beta=0 \\
& D^{2} \Psi_{1}(1)=-i M \operatorname{Re} \Gamma_{0} \\
& \Psi_{1}(0)=D \Psi_{1}(0)=0 .
\end{aligned}
$$

We solved this problem using Maple and obtained a formula for $\Psi_{1}$. This formula is too long to present, but furthermore it is in terms of $\Gamma_{1}$ which is determined by considering the next order problem.

\section{The $O\left(k^{2}\right)$ problem}

In order to determine $\Gamma_{1}$ we only require the $O\left(k^{2}\right)$ terms from equations 2.32 and 2.36 which are respectively

$$
\begin{gathered}
i\left[u_{E}(z)-c_{0}\right] \phi_{1}-i c_{1} \phi_{0}=\frac{1}{P e_{b}}\left(D^{2} \phi_{2}-\phi_{0}\right), \quad 0<z<1 \\
i\left(\frac{\Phi_{E}}{2}-c_{0}\right) \Gamma_{1}-i c_{1} \Gamma_{0}+i \Gamma_{E} D \Psi_{1}=-\frac{\Gamma_{0}}{P e_{s}}-\frac{1}{P e_{b}} D \phi_{2}, \quad \text { at } \quad z=1 .
\end{gathered}
$$

Integrating equation 2.53 leads to

$$
\frac{1}{P e_{b}} D \phi_{2}=\left(\frac{1}{P e_{b}}-i c_{1}\right) \phi_{0} z+I(z)
$$

where $I(z)=i \int\left(u_{E}(z)-c_{0}\right) \phi_{1}(z) d z$, which is then substituted into equation 2.54 to give

$$
i\left(\frac{\Phi_{E}}{2}-c_{0}\right) \Gamma_{1}-i c_{1} \Gamma_{0}+i \Gamma_{E} D \Psi_{1}(1)=-\frac{\Gamma_{0}}{P e_{s}}-\left(\frac{1}{P e_{b}}-i c_{1}\right) \phi_{0}-I_{1}(1)
$$

We next substitute the current expression for $\Psi_{1}$ into this equation and solve for $\Gamma_{1}$. This is then put back into the expression for $\Psi_{1}$ which then contains the control parameters and also $c_{1}$. This is then inserted into equation 2.51 which can finally be solved for $c_{1}$ to give a lengthy formula in terms of the control parameters of the problem. It turns out that $\operatorname{Im}\left(c_{1}\right)$ is not identically equal to zero, and for a given set of parameters, if positive indicates that the perturbation is amplified, while if negative indicating that the perturbation is damped. 


\section{Neutral stability relation}

In order to analyize the effect of the parameters on the stability of the flow we consider $\operatorname{Im}\left(c_{1}\right)=0$, which gives the state of neutral stability and describes the threshold between stability and instability. Specifically, we want to solve the neutral stability relation for the Reynolds number to get the critical value, $R e_{\text {crit }}$, for the onset of instability in terms of the other control parameters. Since Re measures the effect of inertia which plays a destabilizing role, we know that the flow is stable if $R e<R e_{\text {crit }}$ and unstable otherwise.

Now, it turns out that some of our current parameters, namely $M, W e, P e_{s}$, and $P e_{b}$ are implicitly dependent on $R e$. To make this dependence explicit we introduce the new parameters $M a, K a, S c_{s}$ and $S c_{b}$ related to the previous parameters by

$$
\begin{aligned}
& W e=\left(\frac{3}{\sin \beta}\right)^{1 / 3} \frac{\gamma\left(\Gamma_{E}\right)}{\gamma(0)} \frac{K a}{R e^{5 / 3}} \text {, where } K a=\gamma(0)\left(\frac{\rho_{0}}{\mu^{4} g}\right)^{\frac{1}{3}} \text { is the Kapitza number } \\
& M=\left(\frac{3}{\sin \beta}\right)^{1 / 3} \frac{M a}{R e^{5 / 3}} \text {, where } M a=-\left.\frac{K a}{\gamma(0)} \frac{d \gamma}{d \Gamma}\right|_{\Gamma=\Gamma_{E}} \text { is the Marangoni number }
\end{aligned}
$$

$P e_{b}=R e S c_{b}$, where $S c_{b}=\frac{\mu}{\rho_{0} D_{b}}$ is the Schmidt number in the bulk and $P e_{S}=R e S c_{s}$, where $S c_{s}=\frac{\mu}{\rho_{0} D_{s}}$ is the Schmidt number on the surface of the fluid. In terms of the new parameters, the neutral stability relation can be expressed as

$$
C_{1}\left(R e^{1 / 3}\right)^{6}+C_{2}\left(R e^{1 / 3}\right)^{3}+C_{3}\left(R e^{1 / 3}\right)+C_{4}=0
$$

where

$C_{1}=S c_{b}\left(\Phi_{E} \xi_{s}+3 \alpha-3 \xi_{s}\right) S c_{s} k_{s}\left(-4158 S c_{b} \alpha^{4} \gamma_{E} \phi_{0}^{3} \xi_{s}-5238 \Phi_{E} S c_{b} \alpha^{3} \gamma_{E} \phi_{0}^{2} \xi_{s}-294 \Phi_{E} S c_{b} \alpha^{3} \phi_{0}^{3} \xi_{s}+\right.$ $4158 S c_{b} \alpha^{4} \phi 0^{3} \xi_{s}-6048 \alpha^{4} \gamma_{E} \phi_{0}^{3} \xi_{s}-1626 \Phi_{E}^{2} S c_{b} \alpha^{2} \gamma_{E} \phi_{0} \xi_{s}-522 \Phi_{E}^{2} S c_{b} \alpha^{2} \phi_{0}^{2} \xi_{s}+5049 \Phi_{E} S c_{b} \alpha^{3} \phi_{0}^{2} \xi_{s}-10098 \Phi_{E} \alpha^{3} \gamma_{E} \phi_{0}^{2} \xi_{s}+$ $2016 \Phi_{E} \alpha^{3} \phi_{0}^{3} \xi_{s}-567 S c_{b} \alpha^{4} \gamma_{E} \phi_{0}^{2}-882 S c_{b} \alpha^{4} \phi_{0}^{3}+567 S c_{b} \alpha^{3} \gamma_{E} \phi_{0}^{2} \xi_{s}+882 S c_{b} \alpha^{3} \phi_{0}^{3} \xi_{s}+6048 \alpha^{4} \phi_{0}^{3} \xi_{s}-228 \Phi_{E}^{3} S c_{b} \alpha \phi_{0} \xi_{s}+$ $1485 \Phi_{E}^{2} S c_{b} \alpha^{2} \phi_{0} \xi_{s}-5478 \Phi_{E}^{2} \alpha^{2} \gamma_{E} \phi_{0} \xi_{s}+3744 \Phi_{E}^{2} \alpha^{2} \phi_{0}^{2} \xi_{s}-423 \Phi_{E} S c_{b} \alpha^{3} \gamma_{E} \phi_{0}-1566 \Phi_{E} S c_{b} \alpha^{3} \phi_{0}^{2}+423 \Phi_{E} S c_{b} \alpha^{2} \gamma_{E} \phi_{0} \xi_{s}+$ $1566 \Phi_{E} S c_{b} \alpha^{2} \phi_{0}^{2} \xi_{s}+10224 \Phi_{E} \alpha^{3} \phi_{0}^{2} \xi_{s}+378 \alpha^{4} \gamma_{E} \phi_{0}^{2}+6048 \alpha^{4} \phi_{0}^{3}-378 \alpha^{3} \gamma_{E} \phi_{0}^{2} \xi_{s}-6048 \alpha^{3} \phi_{0}^{3} \xi_{s}-966 \Phi_{E}^{3} \alpha \gamma_{E} \xi_{s}+$ $2272 \Phi_{E}^{3} \alpha \phi_{0} \xi_{s}-684 \Phi_{E}^{2} S c_{b} \alpha^{2} \phi 0+684 \Phi_{E}^{2} S c_{b} \alpha \phi_{0} \xi_{s}+5616 \Phi_{E}^{2} \alpha^{2} \phi_{0} \xi_{s}+414 \Phi_{E} \alpha^{3} \gamma_{E} \phi_{0}+11232 \Phi_{E} \alpha^{3} \phi_{0}^{2}-$ $414 \Phi_{E} \alpha^{2} \gamma_{E} \phi_{0} \xi_{s}-11232 \Phi_{E} \alpha^{2} \phi_{0}^{2} \xi_{s}+448 \Phi_{E}^{4} \xi_{s}+1008 \Phi_{E}^{3} \alpha \xi_{s}+126 \Phi_{E}^{2} \alpha^{2} \gamma_{E}+6816 \Phi_{E}^{2} \alpha^{2} \phi_{0}-126 \Phi_{E}^{2} \alpha \gamma_{E} \xi_{s}-$ $\left.6816 \Phi_{E}^{2} \alpha \phi_{0} \xi_{s}+1344 \Phi_{E}^{3} \alpha-1344 \Phi_{E}^{3} \xi_{s}\right)$,

$C_{2}=-S c_{s} S c_{b}\left(\Phi_{E} \xi_{s}+3 \alpha-3 \xi_{s}\right)\left(-3564 \alpha^{4} \cot \beta \gamma_{E} k_{s} \phi_{0}^{2} \xi_{s}-3564 \Phi_{E} \alpha^{3} \cot \beta \gamma_{E} k_{s} \phi_{0} \xi_{s}+1188 \Phi_{E} \alpha^{3} \cot \beta k_{s} \phi_{0}^{2} \xi_{s}+\right.$ $3564 \alpha^{4} \cot \beta k_{s} \phi_{0}^{2} \xi_{s}-882 \Phi_{E}^{2} \alpha^{2} \cot \beta \gamma_{E} k_{s} \xi_{s}+1338 \Phi_{E}^{2} \alpha^{2} \cot \beta k_{s} \phi_{0} \xi_{s}+3420 \Phi_{E} \alpha^{3} \cot \beta k_{s} \phi_{0} \xi_{s}-5040 \Phi_{E} \alpha^{2} \cot \beta \gamma_{E} k_{s} \phi_{0} \xi_{s}-$ $432 \alpha^{4} \cot \beta \gamma_{E} k_{s} \phi_{0}+3564 \alpha^{4} \cot \beta k_{s} \phi_{0}^{2}+432 \alpha^{3} \cot \beta \gamma_{E} k_{s} \phi_{0} \xi_{s}-3564 \alpha^{3} \cot \beta k_{s} \phi_{0}^{2} \xi_{s}+364 \Phi_{E}^{3} \alpha \cot \beta k_{s} \xi_{s}+$ $819 \Phi_{E}^{2} \alpha^{2} \cot \beta k_{s} \xi_{s}-2520 \Phi_{E}^{2} \alpha \cot \beta \gamma_{E} k_{s} \xi_{s}+1680 \Phi_{E}^{2} \alpha \cot \beta k_{s} \phi_{0} \xi_{s}-189 \Phi_{E} \alpha^{3} \cot \beta \gamma_{E} k_{s}+4014 \Phi_{E} \alpha^{3} \cot \beta k_{s} \phi_{0}+$ $189 \Phi_{E} * \alpha^{2} \cot \beta \gamma_{E} k_{s} \xi_{s}+1026 \Phi_{E} \alpha^{2} \cot \beta k_{s} \phi_{0} \xi_{s}-15120 \alpha^{4} \phi_{0}^{3}+1120 \Phi_{E}^{3} \cot \beta k_{s} \xi_{s}+1092 \Phi_{E}^{2} \alpha^{2} \cot \beta k_{s}+$ $1428 \Phi_{E}^{2} \alpha \cot \beta k s \xi_{s}-17640 \Phi_{E} \alpha^{3} \phi_{0}^{2}+5040 \Phi_{E} \alpha^{2} \cot \beta k_{s} \phi_{0}-5040 \Phi_{E} \alpha \cot \beta k_{s} \phi_{0} \xi_{s}-5040 \phi_{E}^{2} \alpha^{2} \phi_{0}+3360 \Phi_{E}^{2} \alpha \cot \beta k_{s}-$ $\left.3360 \Phi_{E}^{2} \cot \beta k_{s} \xi_{s}\right)$,

$C_{3}=1260 S M a S c_{b} S c_{s} \alpha k_{s} \phi_{0}\left(\gamma_{E}-1\right) \xi_{s}\left(-18 \alpha^{2} \gamma_{E} \phi_{0} \xi_{s}-6 \Phi_{E} \alpha \gamma_{E} \xi_{s}+6 \Phi_{E} \alpha \phi_{0} \xi_{s}+18 \alpha^{2} \phi_{0} \xi_{s}+4 \Phi_{E}^{2} \xi_{s}+\right.$ $\left.9 \Phi_{E} \alpha \xi_{s}+9 \alpha^{2} \gamma_{E}+18 \alpha^{2} \phi_{0}-9 \alpha \gamma_{E} \xi_{s}-18 \alpha \phi_{0} \xi_{s}+12 \Phi_{E} \alpha-12 \Phi_{E} \xi_{s}\right)$

$$
C_{4}=-\left(5040\left(\Phi_{E} \xi_{s}+3 \alpha-3 \xi_{s}\right)\right) \alpha k_{s} \phi_{0}\left(-3 S c_{b} \alpha \gamma_{E} \xi_{s}+\Phi_{E} S c_{s} \xi_{s}+3 S c_{b} \alpha \xi_{s}+3 S c_{s} \alpha-3 S c_{s} \xi_{s}\right)
$$


and $\gamma_{E}=\gamma\left(\Gamma_{E}\right)$. We note that this is a 6th degree polynomial in $R e^{1 / 3}$, and it can be solved using the Matlab polynomial root finder roots. For all the numerical values that we considered for the parameters we found that the polynomial had only one positive real root. We took this value to be the critical Reynolds number. 


\section{Chapter 3}

\section{Results}

This chapter comprises of an analysis and overview of the Reynolds number for the onset of instability of the equilibrium flow, $R e_{\text {crit }}$, as we determine the effect of the various parameters utilized in the problem. Due to its complicated nature, the formula for $R e_{c r i t}$ is analyzed by examining its plots as a function of the equilibrium surface concentration, $\Gamma_{E}$, for the various fixed values of the other parameters.

Recall that we scale the surface concentration's maximum value to 1 . Which means that, in this discussion, the range of $\Gamma_{E}$ is from 0 to 1 . However, the expression for the equilibrium bulk concentration, $\phi_{E}$ is not defined when $\Gamma_{E}=1$, so we adjust our interval to $[0,0.99]$ to prevent the associated numerical issues.

In order to generate these plots we must specify an equation of state for the surface tension as a function of $\Gamma_{E}$. We employ the equation of state for surface tension proposed by Sheludko [16]:

$$
\gamma(\Gamma)=\gamma_{0}\left[1+\Gamma\left(\Sigma^{1 / 3}-1\right)\right]^{-3}
$$

where $\gamma_{0}$ is the surface tension of the fluid without surfactant and $\Sigma$ is the ratio of this quantity to that corresponding to the maximal packing of surfactants. In this case the Marangoni number in our formulation becomes

$$
M a=3 K a\left(\Sigma^{1 / 3}-1\right)\left[1+\Gamma_{E}\left(\Sigma^{1 / 3}-1\right)\right]^{-4} .
$$

For the basic problem where the surfactants are assumed to be insoluble, it has been shown $[13,17]$ that the resulting Marangoni effect dampens surface waves and thus counteracts the destabilizing effect of inertia. The fluid velocity at the surface of the layer is non-uniform if the surface undulates, and due to the advective transport of surfactant, concentration differences arise along the surface. It turns out that the surfactant concentration is maximized at the crests of the waves and minimized at the troughs. Since surface tension is weakened as the surfactant concentration increases, the Marangoni stresses pull fluid from the crests to the troughs decreasing the amplitude of the surface waves.

We point out that if the density variation parameter, $\alpha$, is set to zero corresponding to the constant density case, our results coincide with those obtained by Karapetsas and Bontozoglou [13] once the 


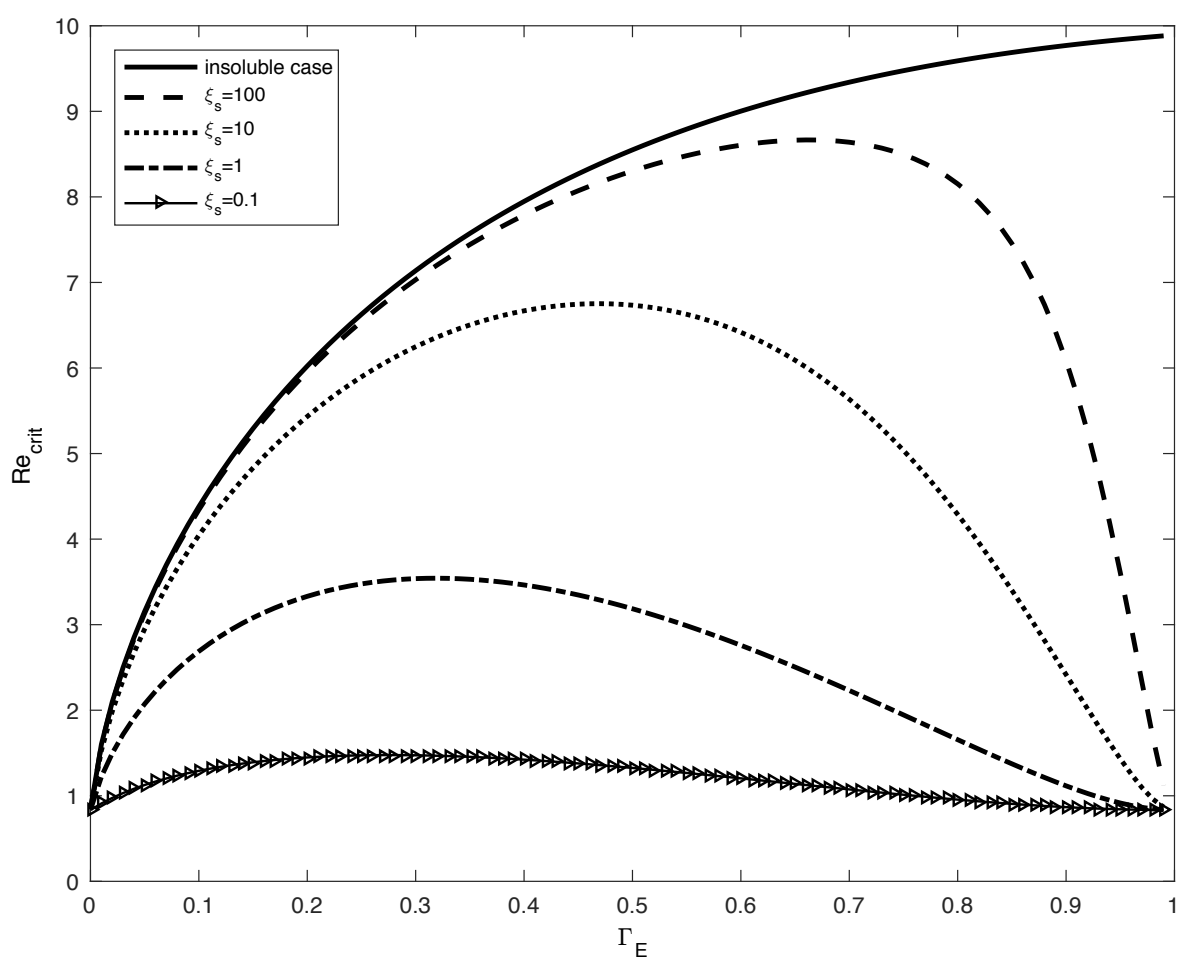

Figure 3.1: The critical Reynolds number as a function of the equilibrium surface concentration for various values of $\xi_{s}$ with $\cot \beta=1, K a=100$ and $\Sigma=2$

difference in scaling is accounted for.

\subsection{Interpretation and discussion of the results}

In Figure 3.1 we show the critical Reynolds number as a function of $\Gamma_{E}$ in the constant density case for various values of $\xi_{s}$. In the insoluble surfactant case $\left(\xi_{s} \rightarrow \infty\right)$ it can be seen that $R e_{c r i t}$ increases with $\Gamma_{E}$, indicating that increasing the equilibrium surfactant concentration stabilizes the flow. It turns out that the leading order term in the amplitude of the surface concentration perturbation is proportional to the equilibrium concentration $\left(\Gamma_{0}=2 \Gamma_{E}\right)$, thus the concentration difference between crests and troughs increases with $\Gamma_{E}$ amplifying the Marangoni stresses and stabilizing the flow. If the surfactant is soluble then the curve exhibits a maximum and increasing the equilibrium concentration destabilizes the flow if the values are sufficiently large. The surfactant flux from the surface into the bulk of the fluid layer is proportional to the concentration on the surface. So the effect is to reduce concentration differences along the surface which diminishes the Marangoni stresses destabilizing the flow. Consequently, it can also be seen that increasing the solubility (decreasing $\xi_{s}$ ) lowers $R e_{c r i t}$ for every value of $\Gamma_{E}$ and decreases the critical value where the maximum occurs.

We now turn to investigating the effect of the density variation with surfactant concentration. The 
relevant parameter is $\alpha$ which measures the rate of change of the fluid density with respect to concentration. We begin by determining the perturbed fluid velocity at the surface of the film $z=1+\tilde{h}$. Denoting this quantity by $v$, and expanding about $z=1$ while discarding nonlinear perturbation terms we obtain

$$
v=u_{E}(1)+\left.\tilde{u}\right|_{z=1} .
$$

If we now introduce the normal modes we can relate the velocity perturbation to that of the surface position and obtain

$$
v=u_{E}(1)+\hat{u}(1) \tilde{h}=u_{E}(1)+D \psi_{0}(1) \tilde{h}+O(k) .
$$

Introducing the expressions for $u_{E}$ and $\psi_{0}$ yields

$$
v=\frac{3}{2}\left(1+\alpha \phi_{E}\right)+\left[3+\frac{3}{2} \alpha\left(\phi_{0}+2 \phi_{E}\right)\right] \tilde{h}+O(k) .
$$

If we consider a reference frame moving with the same velocity as the surface perturbation, i.e. $c_{0}+$ $O(k)=3+\alpha\left(\phi_{0}+3 \phi_{E}\right)+O(k)$, then the fluid velocity at the surface is given by

$$
v-c_{0}=-\frac{3}{2}\left(1+\alpha \phi_{E}\right)-\alpha \phi_{0}+\left[3+\frac{3}{2} \alpha\left(\phi_{0}+2 \phi_{E}\right)\right] \tilde{h}+O(k) .
$$

Since this velocity is negative it is apparent that the wave propagates faster than the fluid flow. Furthermore, the difference is greater at the troughs, where $\tilde{h}$ is negative. So, in a reference frame where the surface disturbance is stationary the fluid velocity is maximized at the troughs and minimized at the crests. Now, since the system is at steady state in the moving reference frame, the surfactant flux (the product of velocity and concentration) is uniform in the streamwise direction. We can conclude then that the surfactant concentration is at a maximum at the crests and a minimum at the troughs of the surface perturbation. This gives rise to the stabilizing Marangoni stresses described above. The dependence on $\alpha$ of the velocity variation from crest to trough is determined by the $\alpha \phi_{0}$ term in the $\tilde{h}$ coefficient, with $\phi_{0}$ itself being a function of $\alpha$. We have obtained a formula for $\phi_{0}$, however this formula is too complicated to analyze. We have however examined its numerical values and have determined that $\alpha \phi_{0}$ is in fact an increasing function of $\alpha$. We thus conclude that increasing $\alpha$ accentuates the velocity variation along the surface resulting in greater concentration differences which strengthens the surface tension stresses and stabilizes the flow.

It should also be pointed out that an increase in fluid density also affects the flow rate. Calculating the flow rate for the equilibrium flow we obtain

$$
q=\int_{0}^{1} u_{E}(z) d z=1+\frac{\alpha \Gamma_{E}}{\xi_{s}\left(1-\Gamma_{E}\right)} .
$$

It is evident that $q$ increases with $\alpha$ which leads to inertial instability. We thus see that increasing $\alpha$ contributes to competing mechanisms affecting the stability of the flow.

In Figure 3.2 we present $R e_{\text {crit }}$ versus $\Gamma_{E}$ curves for different values of $\alpha$. For the smaller values of 


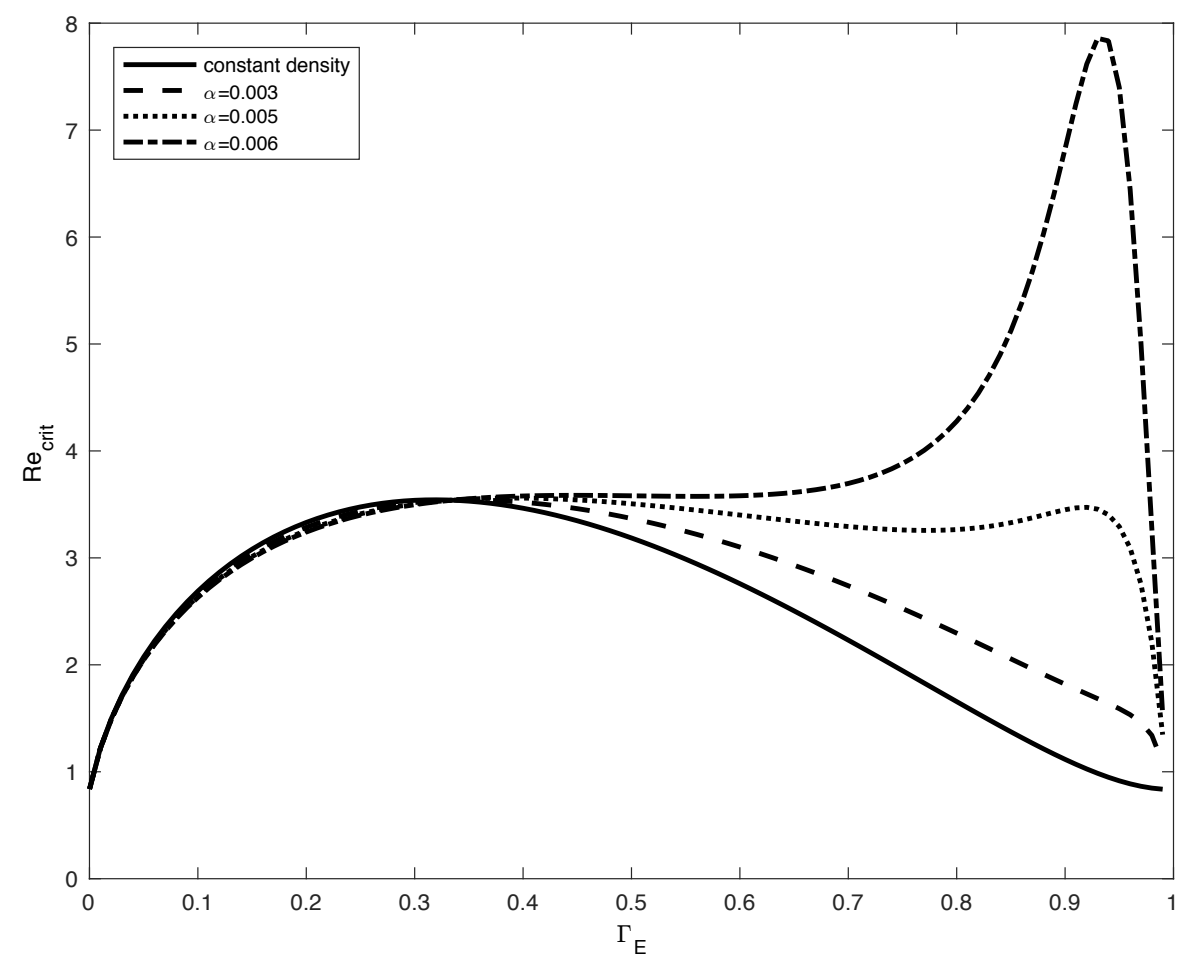

Figure 3.2: The critical Reynolds number as a function of the equilibrium surface concentration for various values of $\alpha$ with $\cot \beta=1, K a=100$ and $\Sigma=2, S c_{b}=700, S c_{s}=700, k_{s}=1$ and $\xi_{s}=1$.

$\Gamma_{E}$ there is a slight decrease in $R e_{c r i t}$ with $\alpha$, while for sufficiently large values of $\Gamma_{E}$ there is a reversal in the destabilizing roles played by density variation. In this case the increase in $R e_{\text {crit }}$ is much more pronounced, especially for the larger values of $\Gamma_{E}$.

In Figure 3.3 we display $R e_{\text {crit }}$ curves for different values of the bulk Schmidt number, $S c_{b}$. It turns out that the surface Schmidt number, $S c_{s}$, has no significant effect on the results. We find that increasing $S c_{b}$ has a similar outcome to increasing $\alpha$ with the exception of the smaller $\Gamma_{E}$ values where there is now no significant effect on the critical Reynolds number. So, increasing $S c_{b}$ has a significant effect on the critical Reynolds number for the larger values of $\Gamma_{E}$ where it acts to increase it. Now, an increase in the Schmidt number can be interpreted as a lowering of the solutal conductivity of the fluid. This has the effect of weakening the transport of surfactant away from the surface. As a result concentration differences are maintained along the surface which has a stabilizing effect.

Figure 3.4 illustrates the effect of the inclination of the film. As $\cot \beta$ is increase the inclination is decreased and a greater Reynolds number is required to generate sufficient inertia to destabilize the flow.

The effect of the rate of change in surface tension with respect to surfactant concentration is presented in Figures 3.5 and 3.6. In our formulation the scaled rate of change is measured by the Marangoni number, $M a$. An increase in this quantity strengthens the Marangoni stresses and stabilizes the flow. 


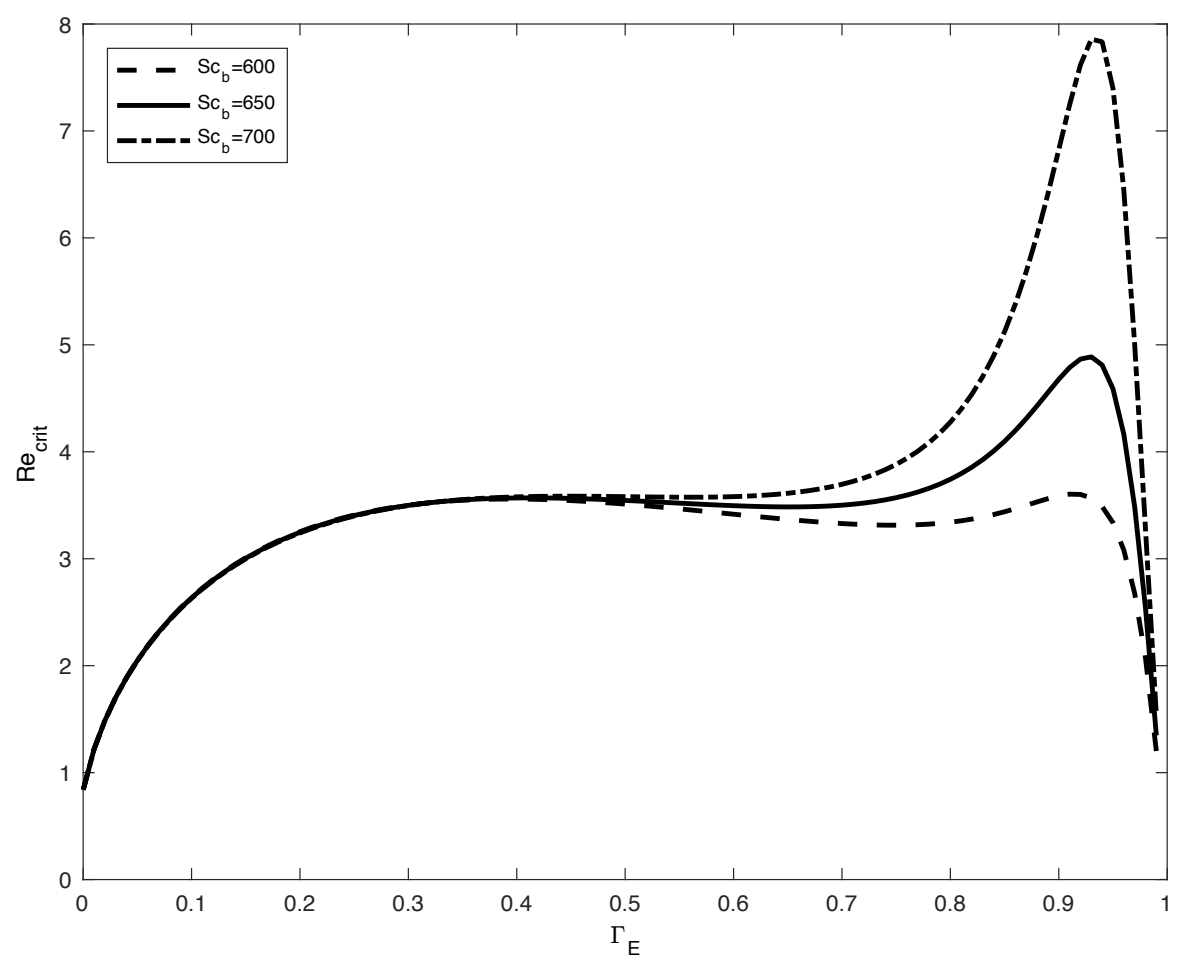

Figure 3.3: The critical Reynolds number as a function of the equilibrium surface concentration for various values of $S c_{b}$ with $\cot \beta=1, K a=100$ and $\Sigma=2, \alpha=0.006, S c_{s}=700, k_{s}=1$ and $\xi_{s}=1$. 


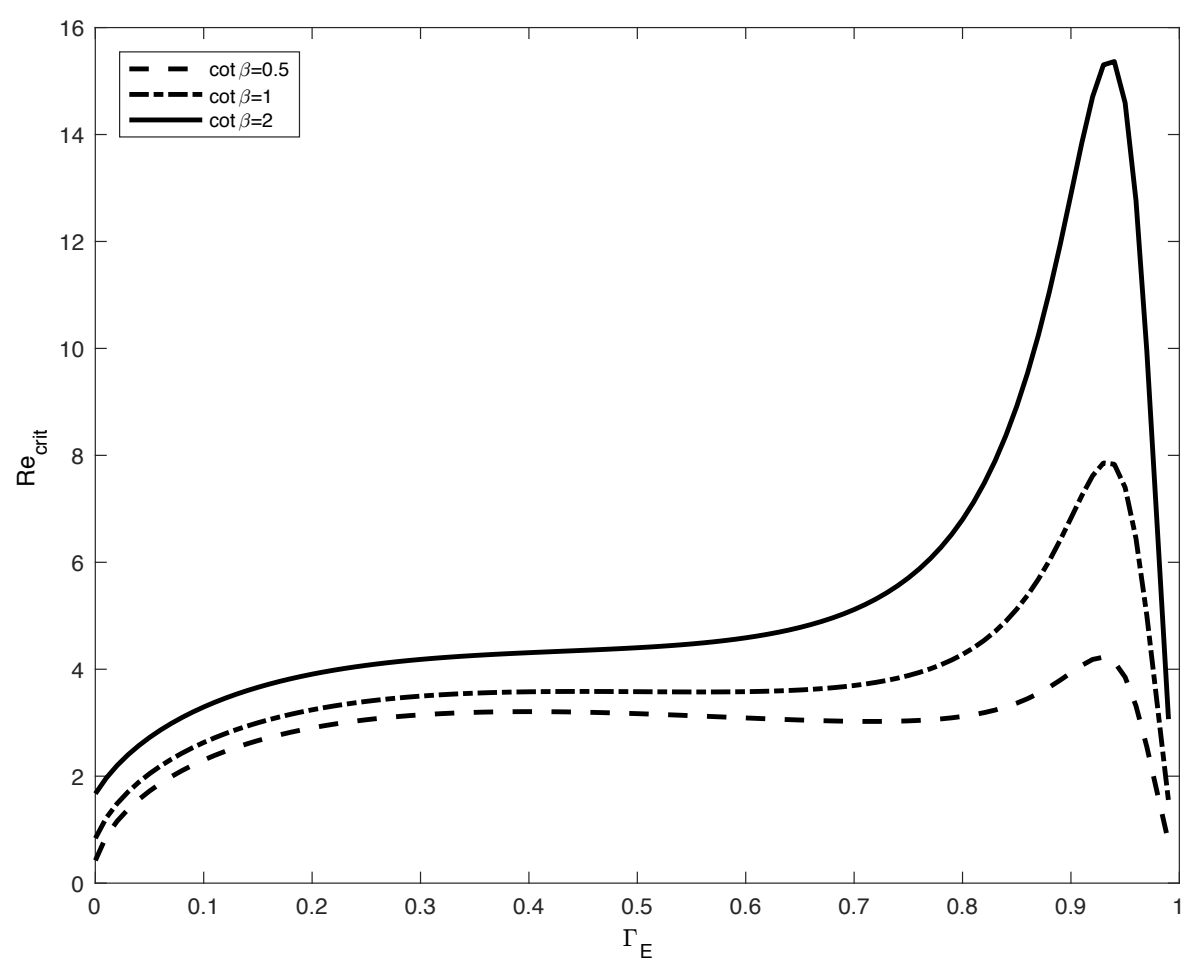

Figure 3.4: The critical Reynolds number as a function of the equilibrium surface concentration for various values of $\cot \beta$ with $K a=100$ and $\Sigma=2, \alpha=0.006, S c_{s}=700, S c_{b}=700, k_{s}=1$ and $\xi_{s}=1$. 


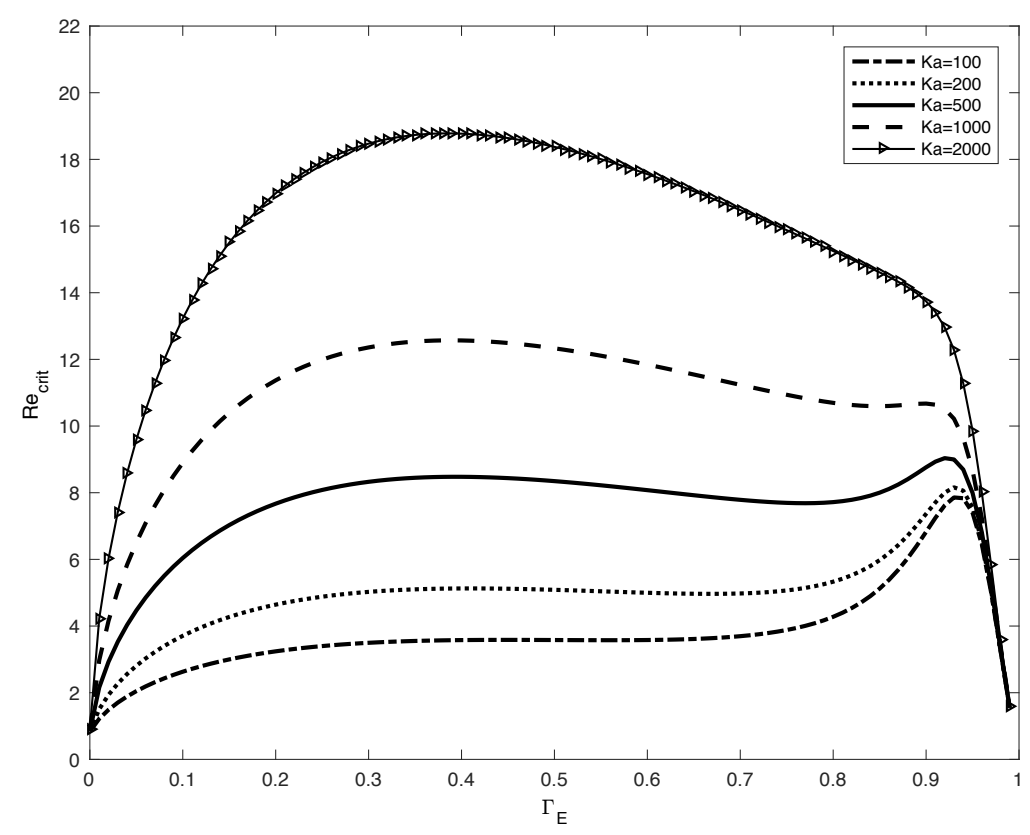

Figure 3.5: The critical Reynolds number as a function of the equilibrium surface concentration for various values of $K a$ with $\cot \beta=1$ and $\Sigma=2, \alpha=0.006, S c_{s}=700, S c_{b}=700, k_{s}=1$ and $\xi_{s}=1$.

For the specific equation of state considered here $M a$ is given by the formula in equation 3.1 and for a given equilibrium concentration the parameters are $K a$ and $\Sigma$. Clearly $M a$ increases with $K a$, and the increase in $R e_{\text {crit }}$ with $K a$ is evident in Figure 3.5. Is also interesting to observe that the "spike-like" structure generated by variable density disappears as $K a$ is increased.

The variation of the Marangoni number with $\Sigma$ is much more complicated and depends on the values $\Gamma_{E}$ and $\Sigma$. The effect of varying $\Sigma$ on the critical Reynolds number is illustrated in Figure 3.6. 


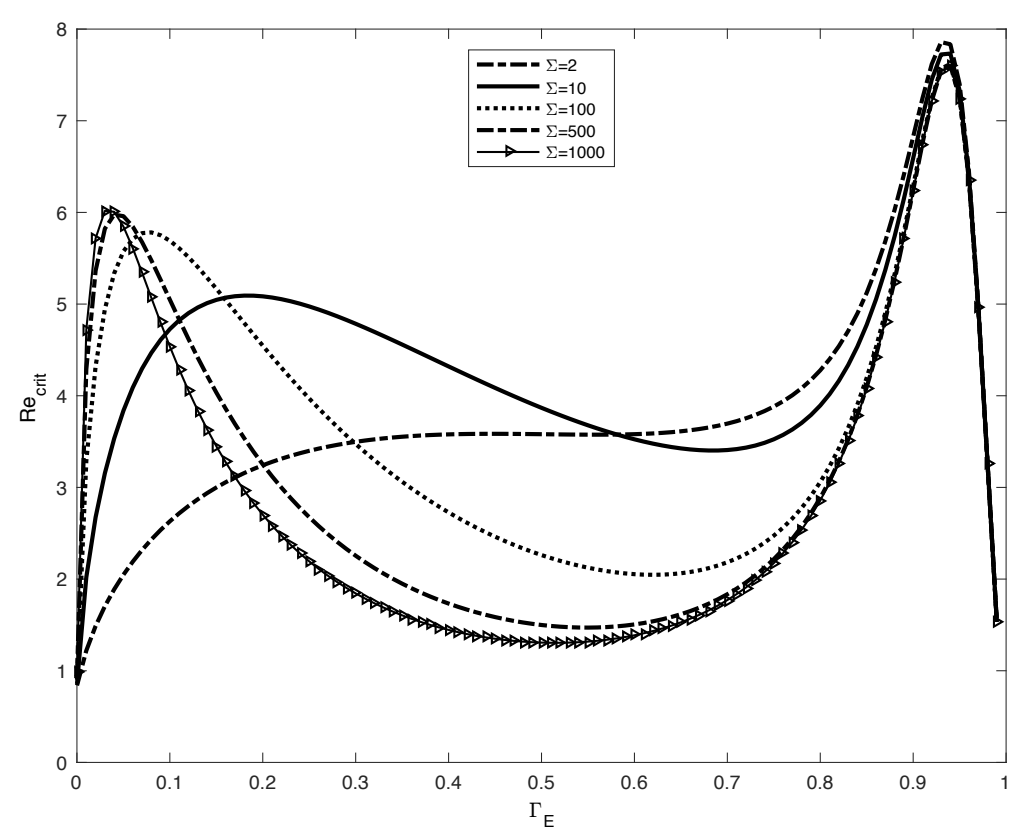

Figure 3.6: The critical Reynolds number as a function of the equilibrium surface concentration for various values of $\Sigma$ with $\cot \beta=1$ and $K a=100, \alpha=0.006, S c_{s}=700, S c_{b}=700, k_{s}=1$ and $\xi_{s}=1$. 


\section{Chapter 4}

\section{Conclusion}

In this thesis we considered the stability of gravity-driven liquid film flow affected by the presence of surfactants. We implemented a mathematical model that captures the solubility of surfactant from the surface into the bulk of the liquid layer, and as novel extension, the model also allows for a variable density for the surfactant solution, which increases with surfactant concentration. A linear equation of state is assumed for the density, with the rate of change with concentration being the control parameter.

We obtained an equilibrium solution to this model corresponding to a steady flow uniform in the streamwise direction. A linear stability analysis was employed to predict the critical conditions for the onset of instability. Specifically, we obtained a formula for neutral stability which was solved for the critical Reynolds number in terms of the other control parameters of the problem, namely, the inclination, the molecular diffusivity of the liquid, the equilibrium concentration of surfactant on the surface of the liquid layer, the rate of change of surface tension with surfactant concentration on the surface, the rate of change of the density with surfactant concentration in the bulk of the layer and the solubility of the surfactant. The principal objective of our study was to draw conclusions regarding the effect of the variable fluid density. It turns out that increasing the rate of variation of the density with surfactant concentration has competing effects on the stability of the flow. On the one hand, increasing the density elevates the flow rate which results in greater inertia which is a contributor to instability. On the other hand, our analysis demonstrates that increasing the variation in density increases the variation in fluid velocity along the surface, which enhances the Marangoni effect leading to a higher instability threshold. Our results indicate that for lower levels of surfactant concentration on the surface, the instability mechanism is slightly more dominant, and increasing the density variation parameter results in lower critical Reynolds numbers. However, if the equilibrium concentration of surfactant absorbed by the surface is sufficiently high, then there is a reversal in the destabilizing role played by density variation.

Our results reveal that the molecular diffusivity of the liquid also has a significant impact on the stability of the flow. In particular, we found that if the equilibrium surface concentration of surfactant is sufficiently high, then increasing the diffusivity of the dissolved surfactant in the bulk of the fluid layer destabilizes the flow. The physical conclusion is that an increase in the molecular conductivity of the 
the liquid enhances the desorption of surfactant from the surface with the effect being greater in regions of higher surface concentration. The results therefore, is the smoothing of concentration variation along the surface, and consequently the weakening of the Marangoni effect which destabilizes the flow. 


\section{References}

1. P.L. Kapitza \& S.P. Kapitza, Wave flow of thin layers of a viscous fluid, Part III: Experimental study of undulatory flow conditions, Soc. Phys. J. Exp. Theor. Phys. 19, 105-120 (1949).

2. T.B. Benjamin, Wave formation in laminar flow down an inclined plane, J. Fluid Mech. 2, 554-574 (1957).

3. C.-S. Yih, Stability of liquid flow down an inclined plane, Phys. Fluids 6, 321-334 (1963).

4. T.B. Benjamin, Effects of surface contamination on wave formation in falling liquid films, Arch. Mech. Stos. 16, 615-626 (1964).

5. S. Whitaker, Effect of surface active agents on the stability of falling liquid films, Ind. Engng Chem. Fundam. 3, 132-142 (1964).

6. M.G. Blyth \& C. Pozrikidis, Effect of surfactant on the stability of film flow down an inclined plane, J. Fluid Mech. 521, 241-250 (2004).

7. A. Pereira, P.M.J. Trevelyan, U. Thiele \& S. Kalliadasis, Dynamics of a horizontal thin liquid film in the presence of reactive surfactants, Phys. Fluids 19, 112102 (2007).

8. A. Pereira \& S. Kalliadasis, Dynamics of a falling film with solutal Marangoni effect, Phys. Rev. E 78, 036312 (2008).

9. R.E. Emmert \& R.L. Pigford, A study of gas absorption in falling liquid films. Chem. Engng Prog. 50 (2), 8793 (1954).

10. C. Stirba \& D. Hurt, Turbulence in falling liquid films. AIChE J. 1, 178184 (1955).

11. S. Tailby \& S. Portalski, The optimum concentration of surface active agents for the suppression of ripples. Trans. Inst. Chem. 39, 328336 (1961).

12. G. Karapetsas \& V. Bontozoglou, The primary instability of falling films in the presence of soluble surfactants, J. Fluid Mech. 729, 123-150 (2013).

13. G. Karapetsas \& V. Bontozoglou, The role of surfactants on the mechanism of long-wave instability in liquid film flows, J. Fluid Mech. 741, 139-155 (2014). 
14. J.H. Spurk \& N. Aksel, Fluid Mechanics (2nd edition) (2008) Springer, Berlin, Germany.

15. A. Pereira \& S. Kalliadasis, On the transport equation for an interfacial quantity, The European Physical Journal Applied Physics 44 (2), 211-214 (2011).

16. A. Sheludko, Thin liquid films, Adv. Colloid Interface Sci. 1, 391-464 (1967).

17. H. Wei, Effect of surfactant on the long-wave instability of a shear-imposed liquid flow down an inclined plane. Phys. Fluids 17, 012103 (2005). 